\title{
Chemically-amplified EUV resists approaching 11 nm half-pitch
}

\author{
Zuhal Tasdemir $^{\mathrm{a}, *}$, Michaela Vockenhuber ${ }^{\mathrm{a}}$, Iacopo Mochi ${ }^{\mathrm{a}}$, Karen Garrido Olvera ${ }^{\mathrm{b}}$, Marieke \\ Meeuwissen $^{\mathrm{b}}$, Oktay Yildirim ${ }^{\mathrm{b}}$, Rik Hoefnagels ${ }^{\mathrm{b}}$, Gijsbert Rispens ${ }^{\mathrm{b}}$, Rolf Custers ${ }^{\mathrm{b}}$, Yasin Ekinci $^{\mathrm{a}}$ \\ ${ }^{a}$ Paul Scherrer Institut, CH-5232 Villigen PSI, Switzerland \\ ${ }^{\mathrm{b}}$ ASML Netherlands B.V., De Run 6501, 5504 DR Veldhoven, the Netherlands \\ *zuhal.tasdemir@psi.ch; Phone: +41 563105807
}

\begin{abstract}
As EUV lithography moves toward high-volume manufacturing phase, one of the key factors determining the throughput and yield is the resist performance, i.e. resolution, sensitivity, and line-edge roughness. At Paul Scherrer Institute (PSI), we extensively work on EUV resist performance issues. For this purpose, we use the PSI's EUV interference lithography (IL) tool in which a coherent beam with $13.5 \mathrm{~nm}$ wavelength is used to produce a well-defined periodic aerial image with virtually $100 \%$ contrast and large depth-of-focus. In this study, we report our recent results on the printability of highresolution lines/spaces pattern down to $11 \mathrm{~nm}$ half-pitch (HP) with a chemically amplified resist (CAR). Although we demonstrate well-resolved $11 \mathrm{~nm}$ HP patterning, further improvement of the CAR's performance in terms of pattern collapse and line-edge roughness is needed. Moreover, these results are achieved with EUV-IL tool which has a fundamentally different aerial image formation than the NXE scanner. Although EUV-IL is a powerful tool to evaluate the resist's ultimate patterning capabilities for early optimization, what is ultimately important is the performance under manufacturing conditions, i.e. at the scanner. Therefore, we address this issue by evaluating the resist performance as a function of the contrast and resolution of the aerial image in a controlled manner, which is possible with the EUV-IL tool. In addition, we compare the performance of the state-of-the-art EUV resists using EUV-IL tool and NXE scanner and attempt to match the data obtained from EUV-IL with the performance of the NXE scanners.
\end{abstract}

Keywords: EUV Interference lithography, image contrast, aerial image, high-resolution patterning, EUV resists, NXE scanner.

\section{INTRODUCTION}

EUV lithography, using $13.5 \mathrm{~nm}$-wavelength, has potential to replace DUV lithography to print the smallest features in the upcoming technology nodes. However the transition from DUV to EUV involves many technological challenges. Resist performance is one of the key challenges, and will remain of a critical importance also at technology nodes beyond the first introduction of EUVL.

Interference lithography (IL) [1], [2] is a cost-effective method to print high-resolution periodic patterns. EUVInterference Lithography (EUV-IL) has been proven to be a valuable alternative for EUV resist testing before the exposure tools become available for HVM. The XIL-II beamline at the Swiss Light Source (SLS) at PSI, is a dedicated beamline for EUV-IL for high-resolution patterning [3]-[10] and it has demonstrated patterning capabilities down to 6 $\mathrm{nm}$ half-pitch (HP) [11], which up to now is still the world record resolution for photon-based lithography. The simple, well-defined, and high-resolution aerial image of EUV-IL makes it a powerful tool for photoresist development. With EUV-IL, it is also possible to pattern several square millimeters in a step and repeat manner, making it a relatively fast technique to get quick feedback on the resists tested, exhibiting a great potential of using EUV-IL for the evaluation of the performance and ultimate resolution limits of different photoresists for future technology nodes.

IL is a relatively simple technique, and it does not require many optical components, and therefore the aerial image is well-defined and the unintended flare is minimized. On the other hand, in NXE scanners, the aerial image formation is more complicated and the resulting aerial image is different from the one of the IL. Although IL provides higher contrast at high resolutions, ultimately, what matters is the resist performance under production conditions, i.e. with the scanner.

Extreme Ultraviolet (EUV) Lithography IX, edited by Kenneth A. Goldberg, Proc. of SPIE Vol. 10583, $105831 \mathrm{~W} \cdot$ ? 2018 SPIE · CCC code: 0277-786X/18/\$18 · doi: 10.1117/12.2299643 
Any resist-related metrology technique such as interference lithography should be aligned and compared with the performance of the EUV scanners to make quantitative prediction.

In this study, we report our recent results on the printability of high resolution lines/spaces pattern down to half-pitch (HP) $11 \mathrm{~nm}$ with a standard EUV chemically amplified resist (CAR). In addition, we address the resist performance as a function of the contrast and resolution of the aerial image. The aim is to match the data obtained from EUV-IL at PSI with the performance of NXE scanners at ASML. For this purpose, after the real interference pattern exposure, we overlay a flat field exposure to simulate different levels of contrast. Moreover, we compare the performance of state-ofthe-art EUV resists with EUV-IL and NXE tools.

\section{EXPERIMENTAL METHODS AND RESULTS}

In this work, EUV exposures were performed with the PSI's EUV-IL tool. Briefly, a spatially coherent incident beam of EUV light is diffracted by linear gratings patterned on a partially transparent membrane [12]. The resulting mutually coherent beams interfere at a certain distance along the beam direction as shown in Fig 1. The aerial image produced by two-beam IL has a period, $P$, that is half of the original mask grating period, $P_{g}$, when first order $(m=l)$ diffracted beams are used (equation 1 ).

$$
P=\frac{\lambda}{2 \sin \theta_{m}}=\frac{P_{g}}{2 m}
$$

Grating-based IL technique offers many advantages such as achromaticity, high resolution, well-defined and pitchindependent contrast aerial image, and large depth-of-focus. The achromaticity means that the contrast and the pitch of the aerial image do not depend on the wavelength as it can be inferred from Eq. 1. Well-defined and pitch-independent aerial image means that it is a sinusoidal pattern of $100 \%$ contrast for all pitches whereas the pitch is defined solely by the grating pitch and the diffraction order. By large depth-of-focus, we mean that the aerial image does not change by the variation of the mask-to-wafer distance, given that the diffraction orders overlap. The gap between the mask and the sample is adjusted to achieve maximum overlapping of the interfering beams. The field size of the patterned area will be minimally affected by small variations between the mask and the wafer.

The mask, consisting of specifically arranged transmission gratings, can be designed to pattern structures with different size and orientation. The gratings are arranged so that the overlap of higher order diffraction beams with the interference patterns is avoided. All areas outside the gratings on the mask are coated with a thick polymer or covered with gold or nickel to completely absorb the zeroth-order beam.

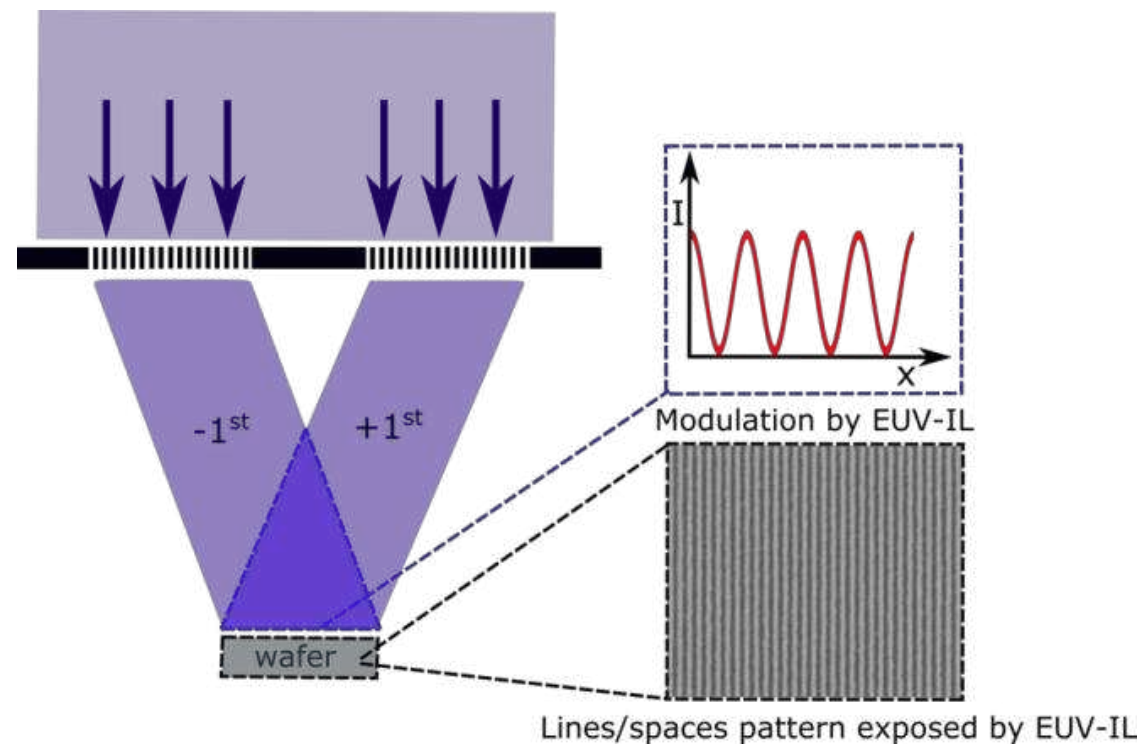

Figure 1. Schematics of EUV-IL setup, showing first diffraction orders that are used for the creation of the aerial image on the wafer. For simplicity only the interfering diffraction orders are shown. 
In this work, we have tested various EUV resists and evaluated their performance. Our aim was to identify resist materials for beyond $13 \mathrm{~nm}$ HP. Although non-CARs can achieve down to $6 \mathrm{~nm}$ HP [11], CARs have been demonstrated down to $12 \mathrm{~nm}$ HP [13]. We have recently achieved $11 \mathrm{~nm}$ HP resolution with an EUV CAR, as shown in Fig. 2. The dose-to-size values are between 57 to $69 \mathrm{~mJ} / \mathrm{cm}^{2}$ for the 16 to $11 \mathrm{~nm} \mathrm{HP}$, respectively. Although further improvement is needed in patterning quality in terms of LER, bridging and partial pattern collapse, all the patterns down to $11 \mathrm{~nm}$ HP are clearly resolved, marking the resolution record of EUV CARs. These results show that CARs hold the potential for beyond $7 \mathrm{~nm}$ nodes with EUVL. Further process optimization and detailed analysis will be performed in future.

Although we demonstrate well-resolved $11 \mathrm{~nm}$ HP patterning, further improvement of the CAR's performance in terms of defects, i.e. pattern collapse and line-edge roughness is needed. Moreover, these results are achieved with EUV-IL tool which has a fundamentally different aerial image formation than the scanner. Although EUV-IL is a powerful tool to evaluate the resist and to check its ultimate patterning capabilities for early optimization of resist formulation and processing, the performance under manufacturing conditions, i.e. NXE scanner, is of a crucial importance.

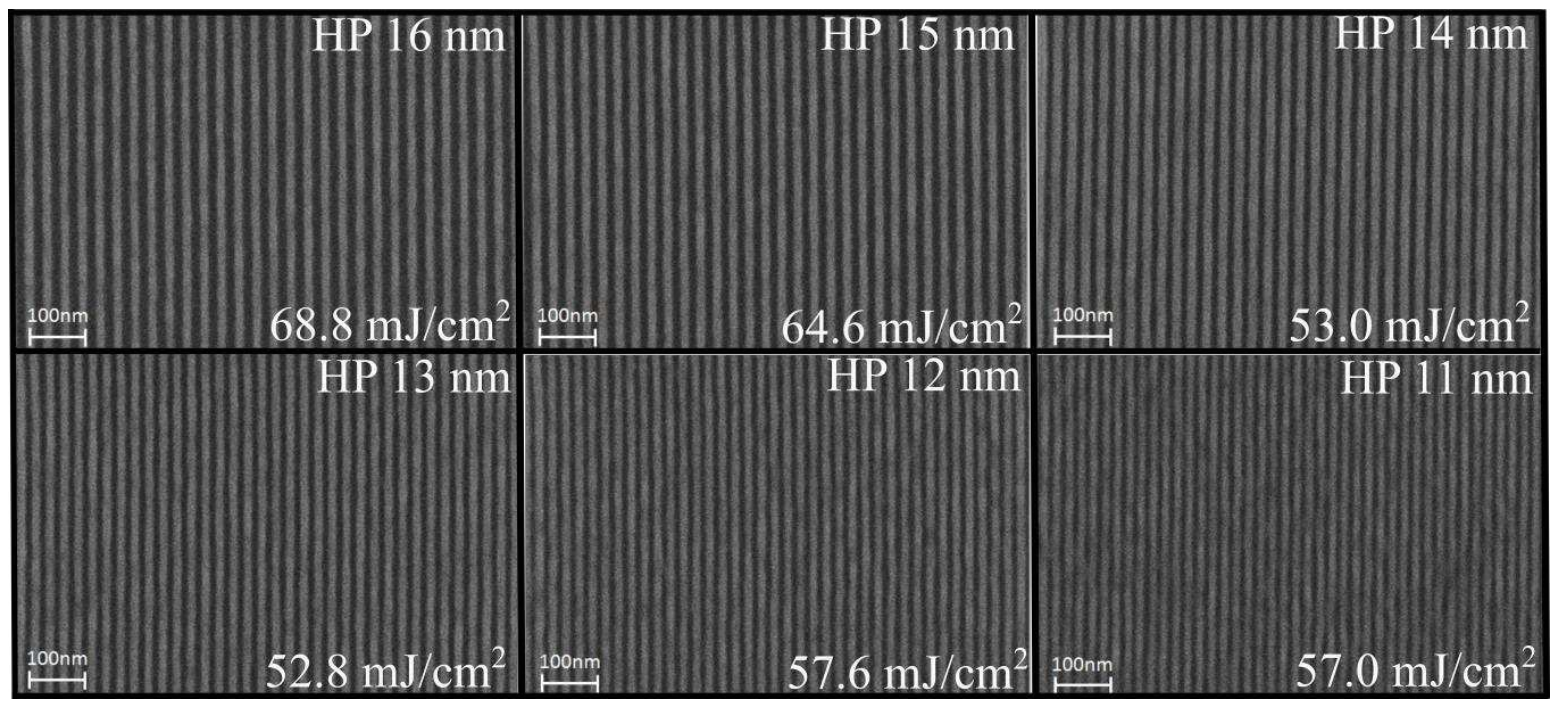

Figure 2. SEM micrographs of the EUV CAR resist resolving from HP $16 \mathrm{~nm}$ down to HP $11 \mathrm{~nm}$.

With EUV-IL, it is possible to perform an assessment of the performance of the advanced EUV materials by probing their maximum resolution limit, as demonstrated above. However, the most important criteria is the performance of the resist under realistic, i.e. reduced contrast, conditions. Moreover, studying the resist contrast by systematically varying the aerial image contrast can provide valuable insights.

In a lithographic tool, the aerial image formed on the wafer level is never ideal and suffers from some contrast loss due to the optics and illumination. The final image in the resist can lose further contrast due to resist and processing. In a scanner, the aerial image formation is fundamentally different than in EUV-IL. For aerial image, Normalized Image Log Slope (NILS) is a useful metric to quantify the aerial image quality and it is used to determine how optical parameters, such as aberrations of the optics, influence the image quality. The NILS of an aerial image generated by a scanner depends on many factors, including the mask, the pattern size, the illumination and the residual flare and aberrations of the scanner optical system, as shown in Fig. 3. When we consider a sinusoidal aerial image created by IL, and calculate the NILS, we get the maximum value of $\pi$, which is also independent of a pitch. This maximum NILS value assumes that there is no contrast loss due to the exposure tool such as incoherent scattering due to the grating roughness and mechanical instabilities. Given the high-resolution of PSI's IL tool, we conclude that such a contrast loss is negligible. In a scanner, NILS is larger than $\pi$ at low resolutions and decreases with decreasing HP.

In the study, we attempt to develop a method to calibrate our EUV-IL exposure tool in order to match the contrast levels with ASML's new generation NXE scanners. From the Fig. 3 we infer that the scanner's aerial image at 22 nm HP with horizontal dipole illumination is close to that of the EUV-IL with $10 \%$ intentionally-added flare background. For 16 nm HP, we predict a similar aerial image with a background of about 15 to $20 \%$ in EUV-IL's aerial image. Line/space patterns with two different periods, $16 \mathrm{~nm} \mathrm{HP}$ and $22 \mathrm{~nm} \mathrm{HP}$, are exposed at both PSI's EUV-IL tool and ASML's NXE scanner, and the results are compared. The method we have used consists of two steps: an interference pattern is exposed with converging first order diffraction beams from two gratings; subsequently, the wafer is moved and a second exposure 
is performed with the diverging first order diffraction beam of one of the gratings, as shown in Fig. 4 below. In this figure, diffracting beams other than first-order are not shown for the sake of simplicity.

EUV-IL exposures are performed on 4" silicon wafers starting from $0 \%$ intentional flare (background) level within a predicted dose range of standard EUV CAR resist, then the dose is adjusted for 5\%, 10\%, 15\%, 20\% and 30\% flare, respectively, in the same manner. After the wafer development, the resulting line/space patterns are inspected by scanning electron microscope (SEM) with an acceleration voltage of $1 \mathrm{kV}$ and a working distance of $3.0 \mathrm{~mm}$ (Supra V55, Zeiss, Germany), Fig. 5. The metrology analysis is carried out by using the commercial software (SUMMIT, Lithometrix, USA).
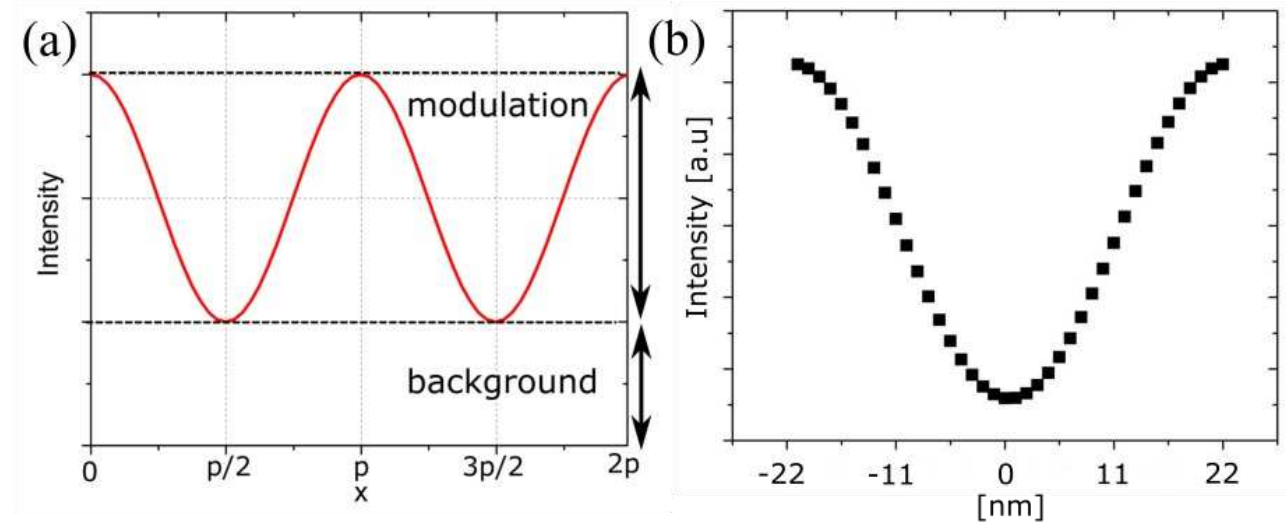

\begin{tabular}{lll}
\hline Flare Levels & NILS Value in $\pi$ & NILS Value \\
\hline $0 \%$ & $1.00 \pi$ & 3.14 \\
$5 \%$ & $0.90 \pi$ & 2.83 \\
$10 \%$ & $0.82 \pi$ & 2.58 \\
$15 \%$ & $0.74 \pi$ & 2.33 \\
$20 \%$ & $0.67 \pi$ & 2.10 \\
$30 \%$ & $0.54 \pi$ & 1.70 \\
\hline
\end{tabular}

\begin{tabular}{ll}
\hline Feature & NILS at BE/BF \\
\hline \hline HP 16 Line/Space Horizontal & 2.25 \\
HP 22 Line/Space Horizontal & 2.53 \\
HP 22 Line/Space Vertical & 2.22 \\
\hline
\end{tabular}

Figure 3. Aerial images and NILS values for (a) EUV-IL and (b) EUV Scanner
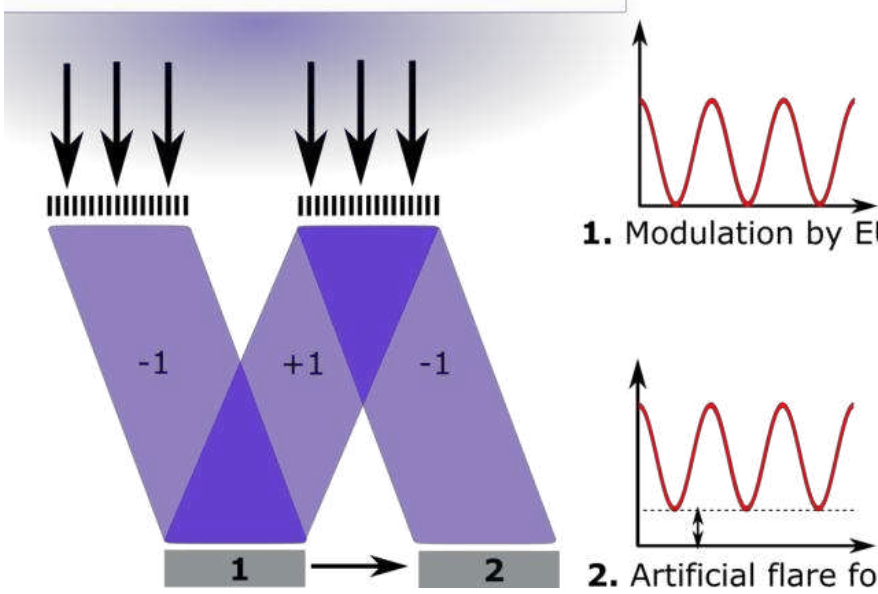

1. Modulation by EUV-IL

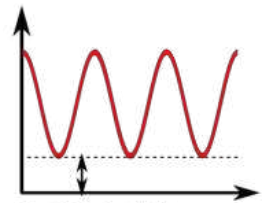

2. Artificial flare formation

Figure 4. The method used to create artificial flare levels in EUV-IL. 

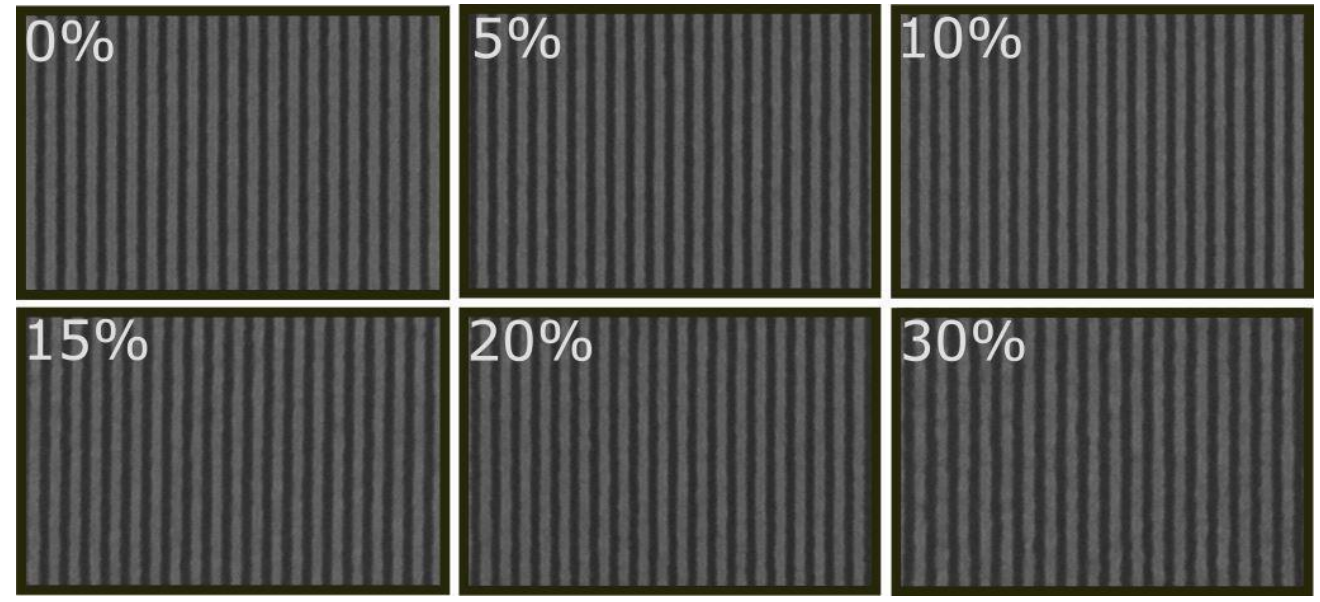

Figure 5. SEM micrographs of lines/spaces patterns (EUV-IL) with 0-30\% intentional flare.

The results are depicted for EUV CAR in Fig. 6 (HP $16 \mathrm{~nm}$ ) and in Fig. 7 (HP $22 \mathrm{~nm}$ ) as CD vs dose for different background levels by EUV-IL and ASML NXE scanner. As it can be seen from the figures, when the contrast level decreases, dose-to-size decreases, the exposure latitude decreases, the slope of the CD-to-dose curves increases, and LWR increases. In general, we conclude that ASML scanner data is similar to the EUV-IL data with the 15\% background level in the case of HP $16 \mathrm{~nm}$ and 10\% background level for HP $22 \mathrm{~nm}$. These background levels are the predicted values for the matched contrast between the IL and the scanner. Dose-to-size values for scanner and IL differ by only $10 \%$ at $22 \mathrm{~nm} \mathrm{HP}$ and $15 \%$ at $16 \mathrm{~nm} \mathrm{HP}$. However, there are significant differences between the results, particularly in the case of HP $16 \mathrm{~nm}$. LWR obtained from the scanner is relatively low and this value seems to match with the EUV-IL data with $0 \%$ contrast level for both HPs. The more apparent differences are in the exposure latitude and the slope of the CD vs dose curves. Currently, we do not have a clear understanding of the root cause. These observations certainly require further investigations in order to achieve a well-matched aerial image and ultimately emulate the NXE scanner with the EUV-IL tool.
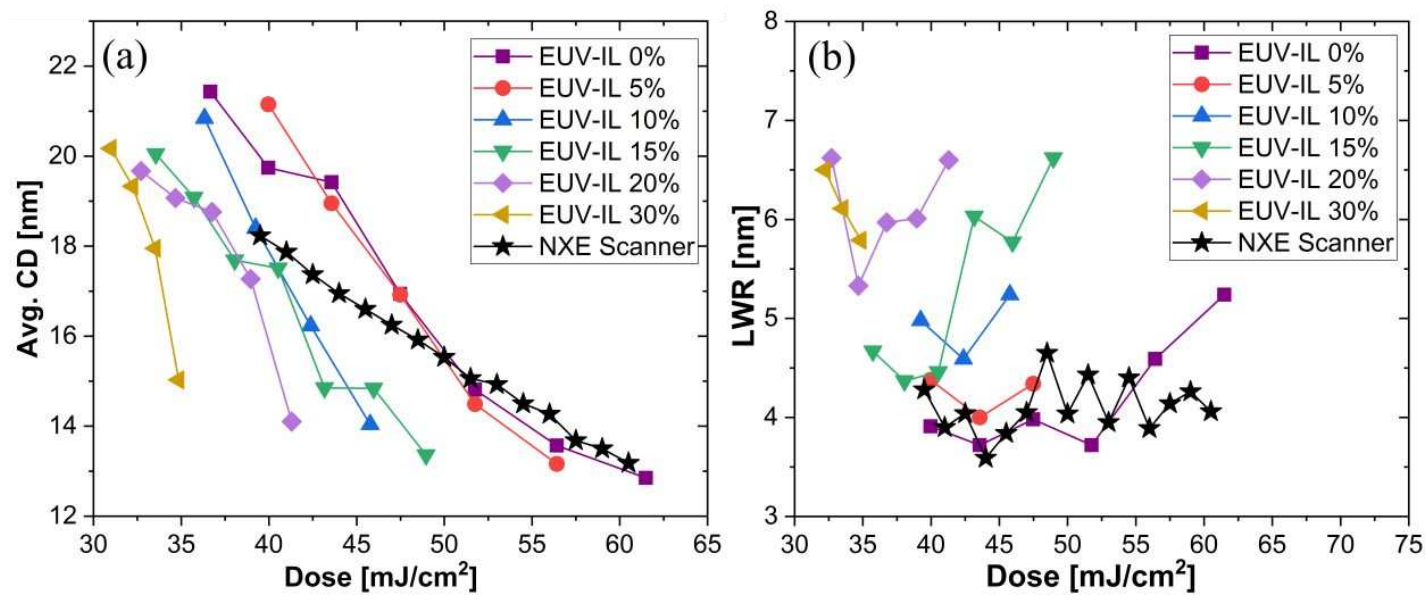

Figure 6. (a) CD vs Dose and (b) LWR vs Dose for different flare levels, by EUV-IL and ASML NXE Scanner for HP $16 \mathrm{~nm}$. 

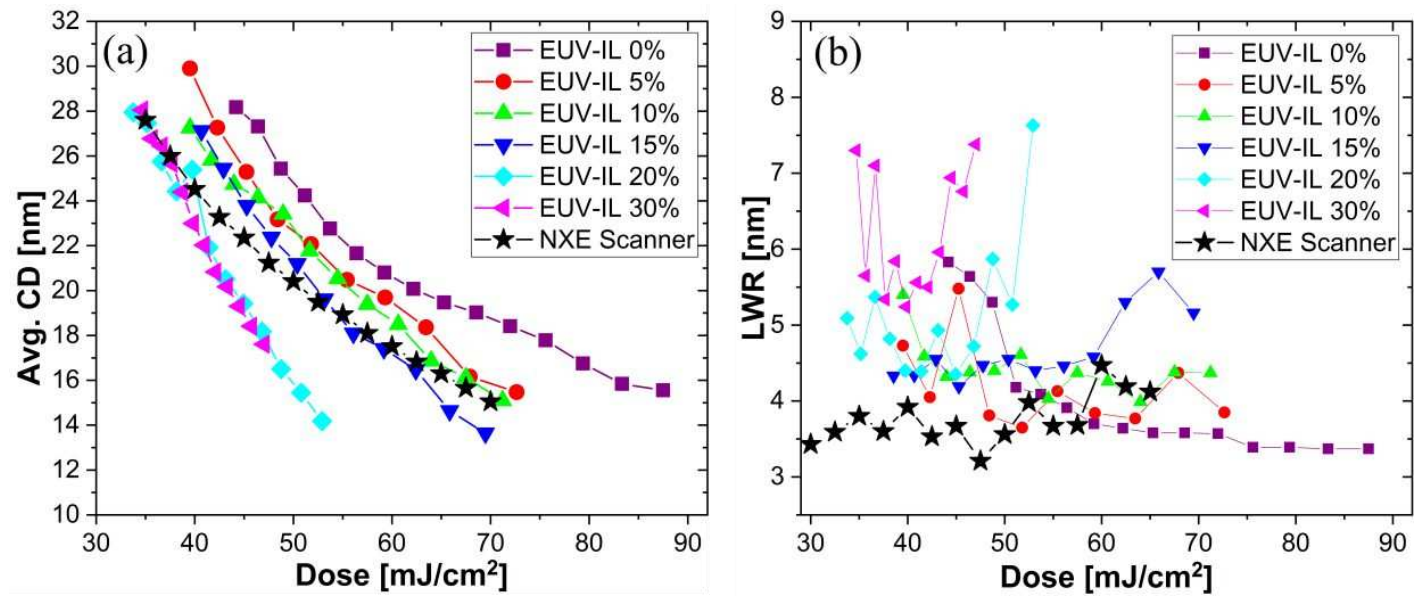

Figure 7. (a) CD vs Dose and (b) LWR vs Dose for different flare levels, by EUV-IL and ASML NXE Scanner for HP $22 \mathrm{~nm}$

\section{CONCLUSIONS AND OUTLOOK}

We showed for the first time the printability of HP $11 \mathrm{~nm}$ with using EUV CAR resist. Well-resolved lines/spaces patterns are obtained and further improvements are needed to solve the defectivity issues such as the pattern collapse and high LER. We developed a method for adjustment of the NILS of EUV-IL in order to match the aerial images of two different lithography tools, i.e. PSI's EUV-IL and ASML's NXE scanner. The method consists of two steps: first order diffraction was used to create an interference pattern, and then another exposure was performed with the diverging first order diffraction beams of one of the gratings to simulate different flare levels. The difference in dose-to-size between these tools is below $15 \%$. The major difference was found to be the slopes of the CD vs dose curves. In the future, we would like to carry out further experiments in order to understand the differences between aerial images of the NXE scanner and contrast-matched IL. This kind of study will help us to develop a method which can reliably predict the resist performance, i.e. dose-to-size, line edge roughness, and exposure latitude, for a given NILS. A method with such a predictive power will enable assessing the performance of a resist without testing it with the scanner and, in particular, estimating the extendibility of the resist for high-NA EUVL for future technology nodes where the aerial image is not available yet but can be calculated.

\section{ACKNOWLEDGEMENTS}

The authors are grateful to Vitaliy Guzenko, Li-Ting Tseng, Dimitrios Kazazis, and Markus Kropf for their invaluable contributions. This project has received funding from the European Union's Horizon 2020 research and innovation programme under the Marie Skłodowska-Curie grant agreement No. 701647.

\section{REFERENCES}

[1] H. H. Solak, "A new beamline for EUV lithography research," in AIP Conference Proceedings, 2000, vol. 521, pp. 99-103.

[2] a Yen, E. H. Anderson, R. a Ghanbari, M. L. Schattenburg, and H. I. Smith, "Achromatic holographic configuration for 100-nm-period lithography.," Appl. Opt., vol. 31, no. 22, pp. 4540-4545, Aug. 1992.

[3] N. Mojarad, D. Fan, J. Gobrecht, and Y. Ekinci, "Broadband interference lithography at extreme ultraviolet and soft x-ray wavelengths.," Opt. Lett., vol. 39, no. 8, p. 2286, 2014.

[4] Y. Ekinci and M. Vockenhuber and B. Terhalle and M. Hojeij and L. Wang and T. R. Younkin, "Evaluation of resist performance with EUV interference lithography for sub-22 nm patterning," in Proc. of SPIE- The International Society for Optical Engineering, 2012, p. 83220W.

[5] E. B. and R. F. and D. F. and T. S. K. and M. V. and Y. Ekinci, "SnOx high-efficiency EUV interference lithography gratings towards the ultimate resolution in photolithography," Microelectron. Eng., vol. 155, no. 44, pp. 44-49, 2016. 
[6] N. Mojarad and J. Gobrecht and Y. Ekinci, "Interference lithography at EUV and Soft X-ray wavelengths: Principles, methods, and applications," Microelectron. Eng, vol. 143, pp. 55-63, 2015.

[7] A. Langner and H.H. Solak and R. Gronheid and E. van Setten and V. Auzelyte and Y. Ekinci and K. van Ingen Schenau and K. Feenstra, "Progress in EUV resist performance," in Proc. of SPIE Extreme UV Lithography, 2010, p. 76362X1-76362X11.

[8] A. Langner and H.H. Solak and R. Gronheid and E. van Setten and V. Auzelyte and Y. Ekinci and K. van Ingen Schenau and K. Feenstra, "Measuring resist-induced contrast loss using EUV interference lithography," in Proc. of SPIE Extreme UV Lithography, 2010, p. 76362X1-76362X11.

[9] Y. E. Elizabeth Buitrago, Seiji Nagahara, Oktay Yildirim, Hisashi Nakagawa, Seiichi Tagawa, Marieke Meeuwissen, Tomoki Nagai, Takehiko Naruoka, Coen Verspaget, Rik Hoefnagels, Gijsbert Rispens, Gosuke Shiraishi, Yuichi Terashita, Yukie Minekawa, Kosuke Yoshihar, "Sensitivity enhancement of chemically amplified resists and performance study using extreme ultraviolet interference lithography," $J$.

Micro/Nanolithography, MEMS, MOEMS, vol. 15, no. 3, p. 33502, 2016.

[10] E. Buitrago et al., "State-of-the-art EUV materials and processes for the 7nm node and beyond," 2017, p. 101430T.

[11] D. Fan and Y. Ekinci, "Photolithography reaches 6 nm half-pitch using EUV light," 2016, p. 97761V.

[12] P. P. Naulleau, C. N. Anderson, and S. F. Horne, "Extreme ultraviolet interference lithography with incoherent light," in Proceedings of SPIE, 2007, vol. 6517, p. 65172T-65172T-8.

[13] E. Buitrago et al., "Sensitivity enhancement of chemically amplified resists and performance study using EUV interference lithography," J. Micro/Nanolithography, MEMS, MOEMS, vol. 15, no. 3, p. 97760Z, Jul. 2016. 